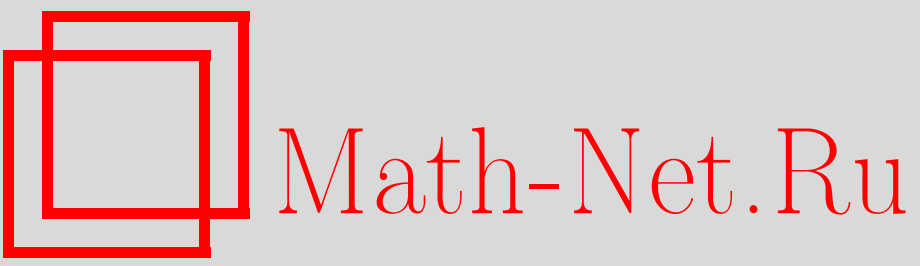

Н. В. Плотникова, Системы линейных дифференциальных уравнений с $\pi$ производной и линейные дифференциальные включения, Матем. сб., 2005, том 196, номер 11, 127-140

DOI: https://doi.org/10.4213/sm1396

Использование Общероссийского математического портала Math-Net.Ru подразумевает, что вы прочитали и согласны с пользовательским соглашением

http://www. mathnet.ru/rus/agreement

Параметры загрузки :

IP: 18.207 .199 .55

26 апреля 2023 г., 18:09:51 
УДК 517.9

\author{
Н.В. Плотникова
}

\title{
Системы линейных дифференциальных уравнений с $\pi$-производной и линейные дифференциальные включения
}

\footnotetext{
В работе построена аппроксимация множества достижимости линейного диффференциального включения с помощью системы линейных дифференциальных уравнений с $\pi$-производной, рассмотрены модельные примеры.

Библиография: 19 названий.
}

\section{Введение}

Рассмотрим линейное дифференциальное включение

$$
\dot{x} \in A(t) x+F(t), \quad x(0) \in X_{0},
$$

где $t \in[0, T], x \in \mathbb{R}^{n}$ - фазовый вектор, $A(t)$ - непрерывная $(n \times n)$-матрица, $F(t)$ непрерывное многозначное отображение $[0, T] \rightarrow \operatorname{comp}\left(\mathbb{R}^{n}\right), X_{0} \in \operatorname{conv}\left(\mathbb{R}^{n}\right)$.

Исследование свойств интегральной воронки включения (1) имеет большое значение в качественной теории и задачах управления линейными системами. В связи с этим многие авторы исследовали свойства множества достижимости [1], [2], а также различные приближенные методы его построения: метод эллипсоидов для линейных систем [3]-[5], асимптотические методы [6], [7], численные методы [8], [9].

В работе [2] была доказана следующая оценка:

$$
R(t) \subset X(t)
$$

где $R(t)$ - множество достижимости включения (1), $X(t)$ - решение уравнения с производной Хукухары [10], соответствуюшего дифференциальному включению (1),

$$
D_{h} X(t)=A(t) X(t)+F(t), \quad X(0)=X_{0} .
$$

Многозначное отображение $X(t)$, исходя из определения производной Хукухары [10], всегда является расширяющимся множеством с увеличением времени. Однако множество достижимости включения (1) может сжиматься и расширяться на различных отрезках времени. В связи с этим в работе рассматривается использование систем дифференциальных уравнений с $\pi$-производной для получения аппроксимации множества достижимости таких систем. 


\section{§1. Понятие $\pi$-производной}

Пространства $\operatorname{comp}\left(\mathbb{R}^{n}\right)$ и $\operatorname{conv}\left(\mathbb{R}^{n}\right)$ не являются линейньми, так как в них отсутствует противоположный элемент и, тем самьм, операция вычитания. В работах [11] и [12] разность в пространстве $\operatorname{conv}\left(\mathbb{R}^{n}\right)$ была введена с помощью вложения его в некоторое линейное пространство. По теореме вложения [13] сушествуют нормированное линейное пространство $\mathscr{B}$ и изометрическое отображение $\pi(\cdot): \operatorname{conv}\left(\mathbb{R}^{n}\right) \rightarrow \mathscr{B}$ такие, что $\pi\left(\operatorname{conv}\left(\mathbb{R}^{n}\right)\right)$ - выпуклый конус в $\mathscr{B}$. Кроме того, сложение и умножение на неотрицательный скаляр в $\mathscr{B}$ влечет соответствующие операции в $\operatorname{conv}\left(\mathbb{R}^{n}\right)$.

Для определения пространства $\mathscr{B}$ вводится соотношение эквивалентности в про$\operatorname{cтранстве~} \operatorname{conv}\left(\mathbb{R}^{n}\right) \times \operatorname{conv}\left(\mathbb{R}^{n}\right)$ следующим образом:

$$
(A, B) \sim(C, D), \quad \text { если } A+D=B+C .
$$

Обозначим через $\langle A, B\rangle$ класс эквивалентности, содержащий $(A, B)$. Тогда пространством $\mathscr{B}$ будет факторпространство $\operatorname{conv}\left(\mathbb{R}^{n}\right) \times \operatorname{conv}\left(\mathbb{R}^{n}\right) / \sim$. В пространстве $\mathscr{B}$ введем операции сложения и умножения на скаляр

$$
\begin{aligned}
& \langle A, B\rangle+\langle C, D\rangle=\langle A+C, B+D\rangle, \\
& \alpha\langle A, B\rangle= \begin{cases}\langle\alpha A, \alpha B\rangle, & \alpha \geqslant 0, \\
\langle\alpha|B,| \alpha \mid A\rangle, & \alpha<0 .\end{cases}
\end{aligned}
$$

Пространство $\mathscr{B}$ является линейньм.

Вложение $\pi(\cdot): \operatorname{conv}\left(\mathbb{R}^{n}\right) \rightarrow \mathscr{B}$ определяется следующим образом:

$$
\pi(A)=\langle A, 0\rangle, \quad A \in \operatorname{conv}\left(\mathbb{R}^{n}\right),
$$

т.е. $\langle A, 0\rangle$ есть класс эквивалентности $\left\{(A+D, D) \mid A, D \in \operatorname{conv}\left(\mathbb{R}^{n}\right)\right\}$.

Метрика и норма в пространстве $\mathscr{B}$ определяется по формулам

$$
\begin{gathered}
\rho(\langle A, B\rangle,\langle C, D\rangle)=h(A+D, C+B), \\
\|\langle A, B\rangle\|=\rho(\langle A, B\rangle,\langle 0,0\rangle) .
\end{gathered}
$$

Пространство $\mathscr{B}$ не является полным метрическим пространством [14], но острый выпуклый замкнутый конус, соответствующий полному метрическому пространству $\operatorname{conv}\left(\mathbb{R}^{n}\right)[15]$, является полным [14].

Пусть $A, B \in \operatorname{conv}\left(\mathbb{R}^{n}\right)$. Разностью множеств $A$ и $B$ называется элемент пространства $\mathscr{B}$, равный $\langle A, B\rangle$. Так как пространство $\mathscr{B}$ является линейным, то данная разность обладает всеми свойствами разности в линейных пространствах.

В обшем случае данная разность двух множеств из $\operatorname{conv}\left(\mathbb{R}^{n}\right)$ не будет являться элементом пространства conv $\left(\mathbb{R}^{n}\right)$. Если же существует разность Хукухары $[16],[10]$ двух множеств из пространства $\operatorname{conv}\left(\mathbb{R}^{n}\right)$, то

$$
\langle A, B\rangle=\langle A \underline{h} B, 0\rangle \text {. }
$$

Рассмотрим более подробно случай $n=1$. Элементами пространства $\operatorname{conv}\left(\mathbb{R}^{1}\right)$ являются сегменты. Поэтому каждый класс эквивалентности представим в виде 
$\langle[x, x+\delta], 0\rangle, \delta \geqslant 0$, либо $\langle x,[0, \delta]\rangle, \delta>0$. Эту запись класса эквивалентности будем называть канонической.

Действительно, произвольный элемент $([a, b],[c, d])$ принадлежит классу первого вида, если $b-a \geqslant d-c$ (в этом случае $x=a-c, \delta=(b-a)-(d-c)$ ), или классу второго вида, если $b-a<d-a$ (в этом случае $x=a-c, \delta=(d-c)-(b-a))$.

Будем записывать класс эквивалентности в канонической форме в виде $(x, \delta)$, понимая при этом $\langle[x, x+\delta], 0\rangle$ при $\delta \geqslant 0$ и $\langle x,[0,-\delta]\rangle=\langle 0,[-x,-x-\delta]\rangle$ при $\delta<0$.

Теорема 1. Справедливы следующие утверждения:

a) $(x, \delta)=(y, \Delta) \Leftrightarrow x=y, \delta=\Delta$;

b) $(x, \delta)+(y, \Delta)=(x+y, \delta+\Delta)$;

c) $a(x, \delta)=(a x, a \delta)$ для любого $a \in \mathbb{R}$.

ДокАЗАТЕльство. а) Достаточность очевидна, докажем необходимость.

Пусть $\delta \geqslant 0, \Delta \geqslant 0$. Тогда $(x, \delta)=\langle[x, x+\delta], 0\rangle=\langle[y, y+\Delta], 0\rangle=(y, \Delta)$, откуда следует, что $x=y, \delta=\Delta$.

Пусть $\delta<0, \Delta<0$. Тогда $(x, \delta)=\langle x,[0,-\delta]\rangle=\langle 0,[-x,-x-\delta]\rangle,(y, \Delta)=$ $\langle y,[0,-\Delta]\rangle=\langle 0,[-y,-y-\Delta]\rangle$, откуда следует, что $x=y, \delta=\Delta$.

Пусть $\delta \geqslant 0, \Delta<0$. Тогда $(x, \delta)=\langle[x, x+\delta], 0\rangle,(y, \Delta)=\langle 0,[-y,-y-\Delta]\rangle$, откуда следует, что равенство $(x, \delta)=(y, \Delta)$ невозможно. Случай $\delta<0, \Delta \geqslant 0$ рассматривается аналогично.

b) Пусть $\delta \geqslant 0, \Delta \geqslant 0$. Тогда

$(x, \delta)+(y, \Delta)=\langle[x, x+\delta], 0\rangle+\langle[y, y+\Delta], 0\rangle=\langle[x+y, x+y+\delta+\Delta], 0\rangle=(x+y, \delta+\Delta)$.

Пусть $\delta<0, \Delta<0$. Тогда

$(x, \delta)+(y, \Delta)=\langle x,[0,-\delta]\rangle+\langle y,[0,-\Delta]\rangle=\langle x+y,[0,-\delta-\Delta]\rangle=(x+y, \delta+\Delta)$.

Пусть $\delta \geqslant 0, \Delta<0$. Тогда

$$
\begin{aligned}
(x, \delta)+(y, \Delta) & =\langle[x, x+\delta], 0\rangle+\langle y,[0,-\Delta]\rangle=\langle[x+y, x+y+\delta],[0,-\Delta]\rangle \\
& =\left\{\begin{array}{ll}
\langle[x+y, x+y+\delta-(-\Delta)], 0\rangle, & \delta \geqslant-\Delta, \\
\langle x+y,[0,-\Delta-\delta]\rangle, & \delta<-\Delta,
\end{array}=(x+y, \delta+\Delta) .\right.
\end{aligned}
$$

Случай $\delta<0, \Delta \geqslant 0$ рассматривается аналогично.

c) При $a=0$ утверждение очевидно. Рассмотрим случай $a>0$. Если $\delta \geqslant 0$, то

$$
a(x, \delta)=a\langle[x, x+\delta], 0\rangle=\langle[a x, a x+a \delta], 0\rangle=(a x, a \delta) .
$$

Если $\delta<0$, то

$$
a(x, \delta)=a\langle x,[0,-\delta]\rangle=\langle a x,[0,-a \delta]\rangle=(a x, a \delta) .
$$

Пусть теперь $a<0$. Тогда если $\delta \geqslant 0$, то

$$
\begin{aligned}
a(x, \delta) & =a\langle[x, x+\delta], 0\rangle=\langle 0,|a|[x, x+\delta]\rangle=\langle 0,[|a| x,|a| x+|a| \delta]\rangle \\
& =(-|a| x,-|a| \delta)=(a x, a \delta) .
\end{aligned}
$$

Если $\delta<0$, то

$$
\begin{aligned}
a(x, \delta) & =a\langle x,[0,-\delta]\rangle=\langle|a|[0,-\delta],|a| x\rangle=\langle[-|a| x,-|a| x-|a| \delta], 0\rangle \\
& =(-|a| x,-|a| \delta)=(a x, a \delta) .
\end{aligned}
$$


ТЕОРема 2. Справедливо следующее утверждение:

$$
\lim _{k \rightarrow \infty}\left(x_{k}, \delta_{k}\right)=(x, \delta) \Longleftrightarrow \lim _{k \rightarrow \infty} x_{k}=x, \quad \lim _{k \rightarrow \infty} \delta_{k}=\delta .
$$

ДокаЗАТЕЛЬство. Докажем необходимость. Пусть начиная с некоторого $k_{0}$ все $\delta_{k} \geqslant 0$. Тогда $\left(x_{k}, \delta_{k}\right)=\left\langle\left[x_{k}, x_{k}+\delta_{k}\right], 0\right\rangle$. Пусть также $\delta \geqslant 0$, тогда $(x, \delta)=$ $\langle[x, x+\delta], 0\rangle$. Так как $\lim _{k \rightarrow \infty}\left(x_{k}, \delta_{k}\right)=(x, \delta)$, то

$$
\begin{aligned}
\rho\left(\left(x_{k}, \delta_{k}\right),(x, \delta)\right) & =\rho\left(\left\langle\left[x_{k}, x_{k}+\delta_{k}\right], 0\right\rangle,\langle[x, x+\delta], 0\rangle\right)=h\left(\left[x_{k}, x_{k}+\delta_{k}\right],[x, x+\delta]\right) \\
& =\max \left\{\left\|x_{k}-x\right\|,\left\|x_{k}+\delta_{k}-x-\delta\right\|\right\} \rightarrow 0
\end{aligned}
$$

при $k \rightarrow \infty$. Следовательно, $\lim _{k \rightarrow \infty} x_{k}=x$ и $\lim _{k \rightarrow \infty} \delta_{k}=\delta$.

Если $\delta<0$, то $(x, \delta)=\langle 0,[-x,-x-\delta]\rangle$, а значит,

$$
\begin{aligned}
\rho\left(\left(x_{k}, \delta_{k}\right),(x, \delta)\right) & =\rho\left(\left\langle\left[x_{k}, x_{k}+\delta_{k}\right], 0\right\rangle,\langle 0,[-x,-x-\delta]\rangle\right) \\
& =h\left(\left[x_{k}-x, x_{k}-x+\delta_{k}-\delta\right], 0\right) \\
& =\max \left\{\left\|x_{k}-x\right\|,\left\|x_{k}+\delta_{k}-x-\delta\right\|\right\} \nrightarrow 0,
\end{aligned}
$$

что противоречит условию.

Случай, когда начиная с некоторого $k_{0}$ все $\delta_{k}<0$, рассматривается аналогично.

Пусть сушествует бесконечно много $\delta_{k} \geqslant 0$ и бесконечно много $\delta_{k}<0$. Тогда разделим последовательность номеров $\{k\}$ на две подпоследовательности

$$
\left\{k_{1}: \delta_{k_{1}} \geqslant 0\right\} \quad \text { и } \quad\left\{k_{2}: \delta_{k_{2}}<0\right\} .
$$

Так как любая подпоследовательность сходящейся последовательности сходится, то $\lim _{k_{1} \rightarrow \infty}\left(x_{k_{1}}, \delta_{k_{1}}\right)=(x, \delta)$ и по доказанному

$$
\lim _{k_{1} \rightarrow \infty} x_{k_{1}}=x, \quad \lim _{k_{1} \rightarrow \infty} \delta_{k_{1}}=\delta \geqslant 0,
$$

т.е. для любого $\varepsilon>0$ существует $k_{1}^{0}$ такое, что при $k_{1}>k_{1}^{0}$ справедливы оценки

$$
\left\|x_{k_{1}}-x\right\|<\varepsilon, \quad\left\|\delta_{k_{1}}-\delta\right\|<\varepsilon .
$$

Аналогично получаем, что $\lim _{k_{2} \rightarrow \infty}\left(x_{k_{2}}, \delta_{k_{2}}\right)=(x, \delta)$, а значит,

$$
\lim _{k_{2} \rightarrow \infty} x_{k_{2}}=x, \quad \lim _{k_{2} \rightarrow \infty} \delta_{k_{2}}=\delta \leqslant 0,
$$

т.е. для любого $\varepsilon>0$ существует $k_{2}^{0}$ такое, что при $k_{2}>k_{2}^{0}$ справедливы оценки

$$
\left\|x_{k_{2}}-x\right\|<\varepsilon, \quad\left\|\delta_{k_{2}}-\delta\right\|<\varepsilon .
$$

Таким образом, получаем, что $\delta=0$. Выбирая $k_{0}=\max \left\{k_{1}^{0}, k_{2}^{0}\right\}$, получаем, что $\left\|x_{k}-x\right\|<\varepsilon$ и $\left\|\delta_{k}\right\|<\varepsilon$ при $k>k_{0}$. Следовательно, необходимость доказана.

Достаточность доказьвается аналогично. 
Теорема 3. Имеем

$$
D_{\pi}(x(t), \delta(t))=(\dot{x}(t), \dot{\delta}(t)),
$$

где $D_{\pi}(x(t), \delta(t))-\pi$-производная [11], [12] парьы $(x(t), \delta(t))$.

ДоКАЗАТЕЛЬСТво. Воспользовавшись определением $\pi$-производной и теоремами 1 и 2, получим

$$
\begin{aligned}
D_{\pi}(x(t), \delta(t)) & =\lim _{\Delta \rightarrow 0} \frac{1}{\Delta}[(x(t+\Delta), \delta(t+\Delta))-(x(t), \delta(t))] \\
& =\lim _{\Delta \rightarrow 0} \frac{1}{\Delta}[(x(t+\Delta), \delta(t+\Delta))+(-x(t),-\delta(t))] \\
& =\lim _{\Delta \rightarrow 0} \frac{1}{\Delta}(x(t+\Delta)-x(t), \delta(t+\Delta)-\delta(t)) \\
& =\lim _{\Delta \rightarrow 0}\left(\frac{x(t+\Delta)-x(t)}{\Delta}, \frac{\delta(t+\Delta)-\delta(t)}{\Delta}\right) \\
& =\left(\lim _{\Delta \rightarrow 0} \frac{x(t+\Delta)-x(t)}{\Delta}, \lim _{\Delta \rightarrow 0} \frac{\delta(t+\Delta)-\delta(t)}{\Delta}\right)=(\dot{x}(t), \dot{\delta}(t)),
\end{aligned}
$$

что и требовалось доказать.

ПримеР 1. Рассмотрим линейное дифференциальное включение

$$
\dot{x} \in a x+[-m, m], \quad x(0)=0,
$$

при $m>0$ и различных $a \neq 0$. В работе А. А. Толстоногова [2] рассматривается вопрос о связи между $R$-решением $R(t)$ [1] включения (2) и решением соответствующего уравнения с производной Хукухары

$$
D_{h} X(t)=a X(t)+[-m, m], \quad X(0)=0 .
$$

В работе было показано, что при $a>0$ справедливо равенство

$$
R(t)=X(t)=\frac{e^{a t}-1}{a}[-m, m]
$$

при $t \in[0, T]$. Если же $a<0$, то

$$
R(t)=\frac{e^{a t}-1}{a}[-m, m], \quad X(t)=e^{-a t} R(t) .
$$

Следовательно, $R(t) \subset X(t)$ при $t \in(0, T]$.

Рассмотрим уравнение с $\pi$-производной, соответствуюшее включению (2):

$$
\left\{\begin{array}{l}
D_{\pi}(x, \delta)=a(x, \delta)+(-m, 2 m) \\
(x(0), \delta(0))=(0,0)
\end{array}\right.
$$


На основании теорем 1 и 3 данное уравнение распадается на два линейных неоднородных уравнения

$$
\left\{\begin{array} { l } 
{ \dot { x } = a x - m , } \\
{ x ( 0 ) = 0 , }
\end{array} \quad \left\{\begin{array}{l}
\dot{\delta}=a \delta+2 m, \\
\delta(0)=0 .
\end{array}\right.\right.
$$

Решая их, получаем, что

$$
x(t)=-\frac{m}{a}\left(e^{a t}-1\right), \quad \delta(t)=\frac{2 m}{a}\left(e^{a t}-1\right) .
$$

Таким образом,

$$
\begin{aligned}
(x(t), \delta(t)) & =\left(-\frac{m}{a}\left(e^{a t}-1\right), \frac{2 m}{a}\left(e^{a t}-1\right)\right) \\
& =\left\langle\left[-\frac{m}{a}\left(e^{a t}-1\right), \frac{m}{a}\left(e^{a t}-1\right)\right], 0\right\rangle=\left\langle\frac{e^{a t}-1}{a}[-m, m], 0\right\rangle .
\end{aligned}
$$

Следовательно, $R$-решение включения (2) при всех $a$ совпадает с интегральной воронкой включения (2) и совпадает с решением уравнения с $\pi$-производной (4).

Таким образом, имеет смысл рассмотреть вопрос об аппроксимации $R$-решения линейного дифференциального включения при помощи решения соответствуюшего уравнения с $\pi$-производной.

\section{§ 2. Аппроксимация множества достижимости линейного дифференциального включения}

Рассмотрим линейное дифференциальное включение

$$
\dot{x} \in A(t) x+F(t), \quad x(0) \in X_{0},
$$

где $t \in[0, T], x \in \mathbb{R}^{n}$ - фазовый вектор, $A(t)$ - непрерывная $(n \times n)$-матрица, $F(t)$ непрерывное многозначное отображение $[0, T] \rightarrow \operatorname{comp}\left(\mathbb{R}^{n}\right), X_{0} \in \operatorname{conv}\left(\mathbb{R}^{n}\right)$.

$R$-решение $R(t)$ включения (5) с начальньм условием $R(0)=X_{0}$ записьвается в виде

$$
R(t)=\Phi(t, 0) X_{0}+\int_{0}^{t} \Phi(t, s) F(s) d s
$$

где

$$
\Phi(t, s)=\exp \int_{s}^{t} A(\tau) d \tau,
$$

интеграл от многозначного отображения понимается в смысле Аумана [17].

Пусть отображение $\widetilde{F}(t)=F_{1}(t) \times \cdots \times F_{n}(t)$, где $F_{i}(t)=\left[f_{i}(t), f_{i}(t)+r_{i}(t)\right]$, $i=\overline{1, n}$, и множество $\widetilde{X}_{0}=X_{1}^{0} \times \cdots \times X_{n}^{0}$, где $X_{i}^{0}=\left[x_{i}^{0}, x_{i}^{0}+\delta_{i}^{0}\right], i=\overline{1, n}$, таковы, что $F(t) \subseteq \widetilde{F}(t) \quad(F(t) \supseteq \widetilde{F}(t))$ для всех $t \in[0, T]$ и $X_{0} \subseteq \widetilde{X}_{0}\left(X_{0} \supseteq \widetilde{X}_{0}\right)$.

Поставим в соответствие включению (5) систему линейных дифференциальных уравнений с $\pi$-производной вида

$$
\left\{\begin{array}{l}
D_{\pi}\left(x_{i}, \delta_{i}\right)=\sum_{j=1}^{n} a_{i j}(t)\left(x_{j}, \delta_{j}\right)+\left(f_{i}(t), r_{i}(t)\right), \\
\left(x_{i}(0), \delta_{i}(0)\right)=\left(x_{i}^{0}, \delta_{i}^{0}\right), \quad i=\overline{1, n} .
\end{array}\right.
$$


Система (7) в силу теорем 1 и 3 распадается на две системы линейных дифференциальных уравнений

$$
\begin{aligned}
& \left\{\begin{array}{c}
\dot{x}_{i}=\sum_{j=1}^{n} a_{i j}(t) x_{j}+f_{i}(t), \\
x_{i}(0)=x_{i}^{0}, \quad i=\overline{1, n}
\end{array}\right. \\
& \left\{\begin{array}{c}
\dot{\delta}_{i}=\sum_{j=1}^{n} a_{i j}(t) \delta_{j}+r_{i}(t), \\
\delta_{i}(0)=\delta_{i}^{0}, \quad i=\overline{1, n}
\end{array}\right.
\end{aligned}
$$

решениями которых являются

$$
\begin{aligned}
& x(t)=\left(x_{1}(t), \ldots, x_{n}(t)\right)^{T}=\Phi(t, 0) x_{0}+\int_{0}^{t} \Phi(t, s) f(s) d s, \\
& \delta(t)=\left(\delta_{1}(t), \ldots, \delta_{n}(t)\right)^{T}=\Phi(t, 0) \delta_{0}+\int_{0}^{t} \Phi(t, s) r(s) d s .
\end{aligned}
$$

Пусть

$$
X_{i}(t)=\left\{\begin{array}{l}
{\left[x_{i}(t), x_{i}(t)+\delta_{i}(t)\right] \text { при } \delta_{i}(t) \geqslant 0} \\
{\left[x_{i}(t)+\delta_{i}(t), x_{i}(t)\right] \text { при } \delta_{i}(t)<0}
\end{array}\right.
$$

и $\widetilde{X}(t)=X_{1}(t) \times \cdots \times X_{n}(t)$.

Изучим связь между множествами $R(t)$ и $\widetilde{X}(t)$. По свойству опорных функций [18] имеем

$$
\begin{aligned}
c(R(t), \psi)= & c\left(\Phi(t, 0) X_{0}, \psi\right)+c\left(\int_{0}^{t} \Phi(t, s) F(s) d s, \psi\right) \\
= & c\left(X_{0}, \Phi^{T}(t, 0) \psi\right)+\int_{0}^{t} c\left(F(s), \Phi^{T}(t, s) \psi\right) d s \\
\leqslant & (\geqslant) c\left(\widetilde{X}_{0}, \Phi^{T}(t, 0) \psi\right)+\int_{0}^{t} c\left(\widetilde{F}(s), \Phi^{T}(t, s) \psi\right) d s \\
= & \sum_{i=1}^{n}\left\{\left(x_{i}^{0}+\frac{\delta_{i}^{0}}{2}\right)\left(\Phi^{T}(t, 0) \psi\right)_{i}+\frac{\delta_{i}^{0}}{2}\left|\left(\Phi^{T}(t, 0) \psi\right)_{i}\right|\right\} \\
& +\int_{0}^{t} \sum_{i=1}^{n}\left\{\left(f_{i}(s)+\frac{r_{i}(s)}{2}\right)\left(\Phi^{T}(t, s) \psi\right)_{i}+\frac{r_{i}(s)}{2}\left|\left(\Phi^{T}(t, s) \psi\right)_{i}\right|\right\} d s \\
= & \sum_{i=1}^{n}\left\{\left(x_{i}^{0}+\frac{\delta_{i}^{0}}{2}\right) \sum_{j=1}^{n} \varphi_{j i}(t, 0) \psi_{j}+\frac{\delta_{i}^{0}}{2}\left|\sum_{j=1}^{n} \varphi_{j i}(t, 0) \psi_{j}\right|\right\} \\
& +\sum_{i=1}^{n} \int_{0}^{t}\left\{\left(f_{i}(s)+\frac{r_{i}(s)}{2}\right) \sum_{j=1}^{n} \varphi_{j i}(t, s) \psi_{j}+\frac{r_{i}(s)}{2}\left|\sum_{j=1}^{n} \varphi_{j i}(t, s) \psi_{j}\right|\right\} d s \\
= & I_{1}(t, \psi),
\end{aligned}
$$




$$
\begin{aligned}
c(\widetilde{X}(t), \psi)= & \sum_{i=1}^{n} c\left(X_{i}, \psi_{i}\right)=\sum_{i=1}^{n}\left(\left(x_{i}+\frac{\delta_{i}}{2}\right) \psi_{i}+\frac{\left|\delta_{i}\right|}{2}\left|\psi_{i}\right|\right) \\
= & \sum_{i=1}^{n}\left\{\left(\sum_{j=1}^{n} \varphi_{i j}(t, 0)\left(x_{j}^{0}+\frac{\delta_{j}^{0}}{2}\right)\right.\right. \\
& \left.+\int_{0}^{t} \sum_{j=1}^{n} \varphi_{i j}(t, s)\left(f_{j}(s)+\frac{r_{j}(s)}{2}\right) d s\right) \psi_{i} \\
& \left.+\frac{\left|\psi_{i}\right|}{2}\left|\sum_{j=1}^{n} \varphi_{i j}(t, 0) \delta_{j}^{0}+\sum_{j=1}^{n} \int_{0}^{t} \varphi_{i j}(t, s) r_{j}(s) d s\right|\right\} \\
= & I_{2}(t, \psi) .
\end{aligned}
$$

Соотношение между $I_{1}(t, \psi)$ и $I_{2}(t, \psi)$ непосредственно связано с соотношением между

$$
J_{1}(t, \psi)=\sum_{i=1}^{n}\left(\delta_{i}^{0}\left|\sum_{j=1}^{n} \varphi_{j i}(t, 0) \psi_{j}\right|+\int_{0}^{t}\left(r_{i}(s)\left|\sum_{j=1}^{n} \varphi_{j i}(t, s) \psi_{j}\right|\right) d s\right)
$$

$$
J_{2}(t, \psi)=\sum_{i=1}^{n}\left|\psi_{i}\right|\left|\sum_{j=1}^{n} \varphi_{i j}(t, 0) \delta_{j}^{0}+\sum_{j=1}^{n} \int_{0}^{t} \varphi_{i j}(t, s) r_{j}(s) d s\right| .
$$

Таким образом, доказана

ТЕОРема 4. Пусть для линейного дифференциального включения (5) существуют непрерьвная функция $\widetilde{F}(t)$ такая, что $F(t) \subseteq \widetilde{F}(t) \quad(F(t) \supseteq \widetilde{F}(t))$, и множество $\widetilde{X}_{0}$ такое, что $X_{0} \subseteq \widetilde{X}_{0} \quad\left(X_{0} \supseteq \widetilde{X}_{0}\right), u J_{1}(t, \psi) \leqslant J_{2}(t, \psi)$ $\left(J_{1}(t, \psi) \geqslant J_{2}(t, \psi)\right)$ для всех $\psi \in \mathbb{R}^{n}$ и $t \in[0, T]$. Тогда $R(t) \subseteq \tilde{X}(t)$ $(R(t) \supseteq \widetilde{X}(t))$ для всех $t \in[0, T]$.

СЛЕДСТВИЕ 1. При $n=1$ имеем $F(t)=\widetilde{F}(t), X_{0}=\widetilde{X}_{0} u$

$$
\begin{aligned}
J_{1}(t, \psi) & =\delta_{0}|\varphi(t, 0) \psi|+\int_{0}^{t} r(s)|\varphi(t, s) \psi| d s \\
& \geqslant|\psi|\left|\varphi(t, 0) \delta_{0}+\int_{0}^{t} r(s) \varphi(t, s) d s\right|=J_{2}(t, \psi)
\end{aligned}
$$

для всех $\psi \in R$ и $t \in[0, T]$. Таким образом, $R(t) \supseteq \widetilde{X}(t)$.

ПримеР 2. Рассмотрим дифференциальное включение (5) при $n=2$ и диагональной матрице $\Phi(t, s)$. Пусть отображение $\widetilde{F}(t)$ и множество $\widetilde{X}_{0}$ таковы, что $F(t) \supseteq \widetilde{F}(t)$ и $X_{0} \supseteq \widetilde{X}_{0}$. В этом случае

$$
\begin{aligned}
J_{1}(t, \psi)= & \delta_{1}^{0}\left|\varphi_{11}(t, 0) \psi_{1}\right|+\delta_{2}^{0}\left|\varphi_{22}(t, 0) \psi_{2}\right| \\
& +\int_{0}^{t} r_{1}(s)\left|\varphi_{11}(t, s) \psi_{1}\right| d s+\int_{0}^{t} r_{2}(s)\left|\varphi_{22}(t, s) \psi_{2}\right| d s \\
J_{2}(t, \psi)= & \left|\psi_{1}\right|\left|\int_{0}^{t} r_{1}(s) \varphi_{11}(t, s) d s+\varphi_{11}(t, 0) \delta_{1}^{0}\right| \\
& +\left|\psi_{2}\right|\left|\int_{0}^{t} r_{2}(s) \varphi_{22}(t, s) d s+\varphi_{22}(t, 0) \delta_{2}^{0}\right| .
\end{aligned}
$$


Так как функции $\varphi_{11}(t, s)$ и $\varphi_{22}(t, s)$ знакопостоянны (в противном случае матрица $\Phi(t, s)$ не будет невырожденной), то $J_{1}(t, \psi)=J_{2}(t, \psi)$ для всех $\psi \in \mathbb{R}^{n}$ и всех $t \in[0, T]$. Таким образом, в силу доказанной теоремы $R(t) \supseteq \widetilde{X}(t)$ для всех $t \in[0, T]$. Если $F(t)=\widetilde{F}(t)$ и $X_{0}=\widetilde{X}_{0}$, то $R(t)=\widetilde{X}(t)$.

В случае, когда матрица $\Phi(t, s)$ является обратно диагональной, получается та же оценка.

СлЕДСТвиЕ 2. Пусть матрица $\Phi(t, s)$ обладает следующим свойством: каждые строка и столбец матрицы содержат один ненулевой әлемент. Пусть отображсние $\widetilde{F}(t)$ и множество $\widetilde{X}_{0}$ такови, ито $F(t) \supseteq \widetilde{F}(t)$ и $X_{0} \supseteq \widetilde{X}_{0}$. Тогда $R(t) \supseteq \widetilde{X}(t)$ для всех $t \in[0, T]$.

Очевидно, что в обшем случае нельзя сказать, какая из величин $J_{1}(t, \psi), J_{2}(t, \psi)$ больше.

Теорема 5. Пусть для линейного дифференциального включения (5) для любого $k \in \overline{1, n}$ все $\varphi_{k i}(t, s)$ неотрицательны (неположительны) для всех $i \in \overline{1, n}$ nри $t, s \in[0, T]$ и существуют функиия $\widetilde{F}(t)$ такая, ито $F(t) \subseteq \widetilde{F}(t), u$ множество $\widetilde{X}_{0}$ такое, ито $X_{0} \subseteq \widetilde{X}_{0}$. Тогда $R(t) \subseteq \widetilde{X}(t)$ для всех $t \in[0, T]$.

ДокАЗАТЕЛЬСтво. Воспользуемся теоремой 4. Достаточно проверить выполнение условия $J_{1}(t, \psi) \leqslant J_{2}(t, \psi)$ лишш для $\psi=e_{k}, k=\overline{1, n}$, где $e_{k}$ - единичный орт. В этом случае

$$
\begin{aligned}
& J_{1}\left(t, e_{k}\right)=\sum_{i=1}^{n}\left(\delta_{i}^{0}\left|\varphi_{k i}(t, 0)\right|+\int_{0}^{t} r_{i}(s)\left|\varphi_{k i}(t, s)\right| d s\right) \\
& J_{2}\left(t, e_{k}\right)=\left|\sum_{i=1}^{n} \varphi_{k i}(t, 0) \delta_{i}^{0}+\sum_{i=1}^{n} \int_{0}^{t} \varphi_{k i}(t, s) r_{i}(s) d s\right| .
\end{aligned}
$$

Так как для любого $k \in \overline{1, n}$ все $\varphi_{k i}(t, s)$ неотрицательны (неположительны) для всех $i \in \overline{1, n}$ и всех $0 \leqslant s \leqslant t \leqslant T$, то $J_{1}\left(t, e_{k}\right)=J_{2}\left(t, e_{k}\right)$ для всех $k \in \overline{1, n}$ и всех $t \in[0, T]$.

ЗАмЕчанИЕ 1 . Если в теореме $5 F(t)=\widetilde{F}(t)$ и $X_{0}=\widetilde{X}_{0}$, то $J_{1}\left(t, e_{k}\right)=J_{2}\left(t, e_{k}\right)$ для всех $k \in \overline{1, n}$ и всех $t \in[0, T]$. Таким образом, множество $R(t)$ вписано в множество $\widetilde{X}(t)$.

ПримеР 3. В работе [19] было рассмотрено дифференциальное включение (5) при $n=1$ :

$$
\dot{x} \in a(t) x+F(t), \quad x(0) \in X_{0} .
$$

В случае, когда $a(t) \geqslant 0$, а отображение $F:[0, T] \rightarrow \operatorname{conv}\left(\mathbb{R}^{n}\right)$ измеримо и интегрально ограничено, в [2] было показано, что $R$-решение $R(t)$ включения (10) совпадает с решением уравнения с производной Хукухары

$$
D_{h} X=a(t) X+F(t), \quad X(0)=X_{0} .
$$

Рассмотрим уравнение с $\pi$-производной, соответствующее включению (10):

$$
\begin{gathered}
D_{\pi}(x, \delta)=a(t)(x, \delta)+(f(t), r(t)), \\
(x(0), \delta(0))=\left(x_{0}, \delta_{0}\right) .
\end{gathered}
$$


В этом случае $F(t)=\widetilde{F}(t), X_{0}=\widetilde{X}_{0}$ и

$$
\varphi(t, s)=\exp \int_{s}^{t} a(\tau) d \tau>0
$$

при всех $t, s \in[0, T]$.

Таким образом, в силу теоремы 5 и следствия 1 получим, что

$$
\begin{aligned}
R(t)= & \tilde{X}(t)=\left[x_{0} \exp \left(\int_{0}^{t} a(s) d s\right)+\int_{0}^{t} f(s) \exp \left(\int_{s}^{t} a(\tau) d \tau\right) d s\right. \\
& \left.\left(x_{0}+\delta_{0}\right) \exp \left(\int_{0}^{t} a(s) d s\right)+\int_{0}^{t}(f(s)+r(s)) \exp \left(\int_{s}^{t} a(\tau) d \tau\right) d s\right] .
\end{aligned}
$$

ПримеР 4. Рассмотрим линейное неоднородное включение

$$
\left(\begin{array}{l}
\dot{x}_{1} \\
\dot{x}_{2}
\end{array}\right) \in\left(\begin{array}{cc}
-2 & 1 \\
1 & -2
\end{array}\right)\left(\begin{array}{l}
x_{1} \\
x_{2}
\end{array}\right)+\left(\begin{array}{c}
0 \\
{[0,2] \sin ^{2} t}
\end{array}\right), \quad x_{1}(0)=x_{2}(0)=0 .
$$

Матрицант соответстуюшей однородной системы имеет вид

$$
\Phi(t, s)=\frac{1}{2}\left(\begin{array}{ll}
e^{-(t-s)}+e^{-3(t-s)} & e^{-(t-s)}-e^{-3(t-s)} \\
e^{-(t-s)}-e^{-3(t-s)} & e^{-(t-s)}+e^{-3(t-s)}
\end{array}\right) .
$$

Так как все $\varphi_{i j}(t, s)>0$ при $i, j \in 1,2$ и всех $0 \leqslant s \leqslant t \leqslant T$, то в силу теоремы $5 R$-решение $R(t)$ включения (12) является подмножеством множества $\widetilde{X}(t)$, определяемого системой уравнений с $\pi$-производной

$$
\left\{\begin{array}{l}
D_{\pi}\left(x_{1}, \delta_{1}\right)=-2\left(x_{1}, \delta_{1}\right)+\left(x_{2}, \delta_{2}\right), \\
D_{\pi}\left(x_{2}, \delta_{2}\right)=\left(x_{1}, \delta_{1}\right)-2\left(x_{2}, \delta_{2}\right)+\left(0,2 \sin ^{2} t\right), \\
\left(x_{1}(0), \delta_{1}(0)\right)=(0,0), \\
\left(x_{2}(0), \delta_{2}(0)\right)=(0,0) .
\end{array}\right.
$$

Эта система распадается на две системы линейных уравнений

$$
\left\{\begin{array} { l } 
{ \dot { x } _ { 1 } = - 2 x _ { 1 } + x _ { 2 } , } \\
{ \dot { x } _ { 2 } = x _ { 1 } - 2 x _ { 2 } , } \\
{ x _ { 1 } ( 0 ) = 0 , } \\
{ x _ { 2 } ( 0 ) = 0 , }
\end{array} \quad \left\{\begin{array}{l}
\dot{\delta}_{1}=-2 \delta_{1}+\delta_{2}, \\
\dot{\delta}_{2}=\delta_{1}-2 \delta_{2}+2 \sin ^{2} t \\
\delta_{1}(0)=0, \\
\delta_{2}(0)=0,
\end{array}\right.\right.
$$

решениями которых являются

$$
\begin{gathered}
x_{1}(t) \equiv 0, \quad x_{2}(t) \equiv 0, \\
\delta_{1}(t)=-\frac{2}{5} e^{-t}+\frac{10}{195} e^{-3 t}-\frac{8}{65} \sin 2 t+\frac{1}{65} \cos 2 t+\frac{1}{3}, \\
\delta_{2}(t)=-\frac{2}{5} e^{-t}-\frac{10}{195} e^{-3 t}-\frac{18}{65} \sin 2 t-\frac{14}{65} \cos 2 t+\frac{2}{3} .
\end{gathered}
$$

Таким образом, $\widetilde{X}(t)$ стремится к периодическому решению при $t \rightarrow \infty$, в то время как решение уравнения с производной Хукухары, соответствуюшего включению (12), является расширяющимся множеством при $t \rightarrow \infty$. 


\section{§3. Аппроксимация множества достижимости} линейного импульсного дифференциального включения

Рассмотрим линейное импульсное дифференциальное включение

$$
\begin{gathered}
\dot{x} \in A(t) x+F(t), \quad t \neq \tau_{k}, \quad x(0) \in X_{0}, \\
\left.\Delta x\right|_{t=\tau_{k}} \in B_{k} x+P_{k},
\end{gathered}
$$

где моменты импульсов $\tau_{k}$ таковы, что $0 \leqslant \tau_{1}<\cdots<\tau_{N} \leqslant T, B_{k}-(n \times n)$-матрицы, $P_{k} \in \operatorname{conv}\left(\mathbb{R}^{n}\right)$.

Пусть множество $\widetilde{P}_{k}=P_{1}^{k} \times \cdots \times P_{n}^{k}$, где $P_{i}^{k}=\left[p_{i}^{k}, p_{i}^{k}+q_{i}^{k}\right], i=\overline{1, n}$, таково, что $P_{k} \subseteq \widetilde{P}_{k}\left(P_{k} \supseteq \widetilde{P}_{k}\right)$. Поставим в соответствие включению (13) систему линейных дифференциальных уравнений с $\pi$-производной вида

$$
\left\{\begin{array}{l}
D_{\pi}\left(x_{i}, \delta_{i}\right)=\sum_{j=1}^{n} a_{i j}(t)\left(x_{j}, \delta_{j}\right)+\left(f_{i}(t), r_{i}(t)\right), \quad t \neq \tau_{k}, \\
\left.\Delta\left(x_{i}, \delta_{i}\right)\right|_{t=\tau_{k}}=\sum_{j=1}^{n} b_{i j}^{k}\left(x_{j}, \delta_{j}\right)+\left(p_{i}^{k}, q_{i}^{k}\right), \\
\left(x_{i}(0), \delta_{i}(0)\right)=\left(x_{i}^{0}, \delta_{i}^{0}\right), \quad i=\overline{1, n},
\end{array}\right.
$$

где $\left.\Delta\left(x_{i}, \delta_{i}\right)\right|_{t=\tau_{k}}=\left(\left.\Delta x_{i}\right|_{t=\tau_{k}},\left.\Delta \delta_{i}\right|_{t=\tau_{k}}\right)$.

Эта система распадается на две системы линейных неоднородных импульсных дифференциальных уравнений:

$$
\begin{aligned}
& \left\{\begin{array}{l}
\dot{x}_{i}=\sum_{j=1}^{n} a_{i j}(t) x_{j}+f_{i}(t), \quad t \neq \tau_{k}, \\
\left.\Delta x_{i}\right|_{t=\tau_{k}}=\sum_{j=1}^{n} b_{i j}^{k} x_{j}+p_{i}^{k}, \\
x_{i}(0)=x_{i}^{0}, \quad i=\overline{1, n},
\end{array}\right. \\
& \left\{\begin{array}{l}
\dot{\delta}_{i}=\sum_{j=1}^{n} a_{i j}(t) \delta_{j}+r_{i}(t), \quad t \neq \tau_{k}, \\
\left.\Delta \delta_{i}\right|_{t=\tau_{k}}=\sum_{j=1}^{n} b_{i j}^{k} \delta_{j}+g_{i}^{k}, \\
\delta_{i}(0)=\delta_{i}^{0}, \quad i=\overline{1, n} .
\end{array}\right.
\end{aligned}
$$

Пусть $\widetilde{X}\left(\tau_{k}+0\right)=X_{1}\left(\tau_{k}+0\right) \times \cdots \times X_{n}\left(\tau_{k}+0\right)$, где

$$
\begin{gathered}
X_{i}\left(\tau_{k}+0\right)=\left\{\begin{array}{l}
{\left[x_{i}\left(\tau_{k}+0\right), x_{i}\left(\tau_{k}+0\right)+\delta_{i}\left(\tau_{k}+0\right)\right] \text { при } \delta_{i}\left(\tau_{k}+0\right) \geqslant 0,} \\
{\left[x_{i}\left(\tau_{k}+0\right)+\delta_{i}\left(\tau_{k}+0\right), x_{i}\left(\tau_{k}+0\right)\right] \text { при } \delta_{i}\left(\tau_{k}+0\right)<0,}
\end{array}\right. \\
x_{i}\left(\tau_{k}+0\right)=\sum_{j=1}^{n}\left(e_{i j}+b_{i j}^{k}\right) x_{j}\left(\tau_{k}\right)+p_{i}^{k}, \\
\delta_{i}\left(\tau_{k}+0\right)=\sum_{j=1}^{n}\left(e_{i j}+b_{i j}^{k}\right) \delta_{j}\left(\tau_{k}\right)+q_{i}^{k},
\end{gathered}
$$

$e_{i j}$ - символ Кронекера. 
Изучим связь между множествами $R\left(\tau_{k}+0\right)$ и $\widetilde{X}\left(\tau_{k}+0\right)$ в предположении, что $R\left(\tau_{k}\right) \subseteq \widetilde{X}\left(\tau_{k}\right)\left(R\left(\tau_{k}\right) \supseteq \widetilde{X}\left(\tau_{k}\right)\right)$. По свойству опорных функций имеем

$$
\begin{aligned}
c\left(R\left(\tau_{k}+0\right), \psi\right)= & c\left(\left(E+B_{k}\right) R\left(\tau_{k}\right)+P_{k}, \psi\right)=c\left(R\left(\tau_{k}\right),\left(E+B_{k}\right)^{T} \psi\right)+c\left(P_{k}, \psi\right) \\
\leqslant & (\geqslant) c\left(\widetilde{X}\left(\tau_{k}\right),\left(E+B_{k}\right)^{T} \psi\right)+c\left(\widetilde{P}_{k}, \psi\right) \\
= & \sum_{i=1}^{n}\left\{c\left(X_{i}\left(\tau_{k}\right),\left(\left(E+B_{k}\right)^{T} \psi\right)_{i}\right)+c\left(P_{i}^{k}, \psi_{i}\right)\right\} \\
= & \sum_{i=1}^{n}\left\{\left(x_{i}\left(\tau_{k}\right)+\frac{\delta_{i}\left(\tau_{k}\right)}{2}\right) \sum_{j=1}^{n}\left(e_{j i}+b_{j i}^{k}\right) \psi_{j}+\left(p_{i}^{k}+\frac{q_{i}^{k}}{2}\right) \psi_{i}\right. \\
& \left.+\frac{\left|\delta_{i}\left(\tau_{k}\right)\right|}{2}\left|\sum_{j=1}^{n}\left(e_{j i}+b_{j i}^{k}\right) \psi_{j}\right|+\frac{q_{i}^{k}}{2}\left|\psi_{i}\right|\right\}=I_{k 1}(\psi), \\
c\left(\widetilde{X}\left(\tau_{k}+0\right), \psi\right)= & \sum_{i=1}^{n} c\left(X_{i}\left(\tau_{k}+0\right), \psi_{i}\right) \\
= & \sum_{i=1}^{n}\left\{\left(x_{i}\left(\tau_{k}+0\right)+\frac{\delta_{i}\left(\tau_{k}+0\right)}{2}\right) \psi_{i}+\frac{\left|\delta_{i}\left(\tau_{k}+0\right)\right|}{2}\left|\psi_{i}\right|\right\} \\
= & \sum_{i=1}^{n}\left\{\sum_{j=1}^{n}\left(e_{i j}+b_{i j}^{k}\right)\left(x_{j}\left(\tau_{k}\right)+\frac{\delta_{j}\left(\tau_{k}\right)}{2}\right) \psi_{i}+\left(p_{i}^{k}+\frac{q_{i}^{k}}{2}\right) \psi_{i}\right. \\
& \left.+\frac{1}{2}\left|\sum_{j=1}^{n}\left(e_{i j}+b_{i j}^{k}\right) \delta_{j}\left(\tau_{k}\right)+q_{i}^{k}\right|\left|\psi_{i}\right|\right\}=I_{k 2}(\psi) .
\end{aligned}
$$

Соотношение между $I_{k 1}(\psi)$ и $I_{k 2}(\psi)$ непосредственно связано с соотношением между

$$
J_{k 1}(\psi)=\sum_{i=1}^{n}\left\{\left|\delta_{i}\left(\tau_{k}\right)\right|\left|\sum_{j=1}^{n}\left(e_{j i}+b_{j i}^{k}\right) \psi_{j}\right|+q_{i}^{k}\left|\psi_{i}\right|\right\}
$$

$$
J_{k 2}(\psi)=\sum_{i=1}^{n}\left|\sum_{j=1}^{n}\left(e_{i j}+b_{i j}^{k}\right) \delta_{j}\left(\tau_{k}\right)+q_{i}^{k}\right|\left|\psi_{i}\right| .
$$

Таким образом, доказана

Teopema 6. Пусть $P_{k} \subseteq \widetilde{P}_{k} \quad\left(P_{k} \supseteq \widetilde{P}_{k}\right)$ u $X\left(\tau_{k}\right) \subseteq \widetilde{X}\left(\tau_{k}\right) \quad\left(X\left(\tau_{k}\right) \supseteq \widetilde{X}\left(\tau_{k}\right)\right)$. Тогда если $J_{k 1}(\psi) \leqslant(\geqslant) J_{k 2}(\psi)$ для всех $\psi \in \mathbb{R}^{n}$, mо $R\left(\tau_{k}+0\right) \subseteq \widetilde{X}\left(\tau_{k}+0\right)$ $\left(R\left(\tau_{k}+0\right) \supseteq \widetilde{X}\left(\tau_{k}+0\right)\right)$.

СлЕДСТВИЕ 3. При $n=1$ имеем $P_{k}=\widetilde{P}_{k} u$

$$
J_{k 1}(\psi)=\left|\delta_{i}\left(\tau_{k}\right)\left(1+b_{k}\right) \psi\right|+q_{k}|\psi| \geqslant\left|\left(1+b_{k}\right) \delta_{i}\left(\tau_{k}\right)+q_{k}\right| \cdot|\psi|=J_{k 2}(\psi)
$$

для всех $\psi \in \mathbb{R}$. Таким образом, $R\left(\tau_{k}+0\right) \supseteq \widetilde{X}\left(\tau_{k}+0\right)$.

Teорема 7. Пусть $P_{k} \subseteq \widetilde{P}_{k}$ u $R\left(\tau_{k}\right) \subseteq \widetilde{X}\left(\tau_{k}\right)$. Если для любого $m \in \overline{1, n}$ все $\left(e_{m i}+b_{m i}^{k}\right) \delta_{i}\left(\tau_{k}\right)$ неотрицательны для всех $i \in \overline{1, n}$, то $R\left(\tau_{k}+0\right) \subseteq \widetilde{X}\left(\tau_{k}+0\right)$. 
ДоКАЗАТЕЛЬСТво. Достаточно проверить выполнение условия

$$
J_{k 1}(\psi) \leqslant J_{k 2}(\psi)
$$

лишш для $\psi=e_{m}, m=\overline{1, n}$. В этом случае

$$
\begin{aligned}
& J_{k 1}\left(e_{m}\right)=\sum_{i=1}^{n}\left|\delta_{i}\left(\tau_{k}\right)\left(e_{m i}+b_{m i}^{k}\right)\right|+q_{m}^{k}, \\
& J_{k 2}\left(e_{m}\right)=\left|\sum_{i=1}^{n}\left(e_{m i}+b_{m i}^{k}\right) \delta_{i}\left(\tau_{k}\right)+q_{m}^{k}\right| .
\end{aligned}
$$

Так как для любого $m \in \overline{1, n}$ все $\left(e_{m i}+b_{m i}^{k}\right) \delta_{i}\left(\tau_{k}\right)$ неотрицательны для всех $i \in \overline{1, n}$, то $J_{k 1}\left(e_{m}\right)=J_{k 2}\left(e_{m}\right)$ для всех $m \in \overline{1, n}$.

ЗАмечание 2 . Если в теореме $7 P_{k}=\widetilde{P}_{k}$ и $R\left(\tau_{k}\right)=\widetilde{X}\left(\tau_{k}\right)$, то $J_{k 1}\left(e_{m}\right)=$ $J_{k 2}\left(e_{m}\right)$ для всех $m \in \overline{1, n}$. Таким образом, множество $R\left(\tau_{k}+0\right)$ вписано в множество $\widetilde{X}\left(\tau_{k}+0\right)$. При $n=1$ получим, что $R\left(\tau_{k}+0\right)=\widetilde{X}\left(\tau_{k}+0\right)$.

ПримеР 5. Рассмотрим импульсное дифференциальное включение (13) при $n=1$ :

$$
\begin{array}{cl}
x \in a(t) x+F(t), & t \neq \tau_{k}, \\
\left.\Delta x\right|_{t=\tau_{k}} \in b_{k} x+P_{k}, & x(0) \in X_{0} .
\end{array}
$$

Поставим ему в соответствие линейное уравнение с $\pi$-производной

$$
\begin{gathered}
D_{\pi}(x, \delta)=a(t)(x, \delta)+(f(t), r(t)), \quad t \neq \tau_{k}, \\
\left.\Delta(x, \delta)\right|_{t=\tau_{k}}=b_{k}(x, \delta)+\left(p_{k}, q_{k}\right), \\
(x(0), \delta(0))=\left(x_{0}, \delta_{0}\right)
\end{gathered}
$$

которое распадается на два линейных импульсных дифференциальных уравнения:

$$
\left\{\begin{array} { l } 
{ \dot { x } = a ( t ) x + f ( t ) , \quad t \neq \tau _ { k } , } \\
{ \Delta x | _ { t = \tau _ { k } } = b _ { k } x + p _ { k } , } \\
{ x ( 0 ) = x _ { 0 } , }
\end{array} \quad \left\{\begin{array}{l}
\dot{\delta}=a(t) \delta+r(t), \quad t \neq \tau_{k}, \\
\left.\Delta \delta\right|_{t=\tau_{k}}=b_{k} \delta+q_{k}, \\
\delta(0)=\delta_{0}
\end{array}\right.\right.
$$

В этом случае $F(t)=\widetilde{F}(t)$ и $X_{0}=\widetilde{X}_{0}$. Предположим, что $b_{k} \geqslant-1$ для всех $k \in N$. Проверим выполнение условий теоремы 7 :

$$
\left(1+b_{1}\right) \delta\left(\tau_{1}\right)=\left(1+b_{1}\right)\left(\exp \left(\int_{0}^{\tau_{1}} a(t) d t\right) \delta_{0}+\int_{0}^{\tau_{1}} r(s) \exp \left(\int_{s}^{\tau_{1}} a(t) d t\right) d s\right) \geqslant 0 .
$$

Предположим, что $\left(1+b_{k-1}\right) \delta\left(\tau_{k-1}\right) \geqslant 0$. Тогда

$$
\begin{aligned}
\left(1+b_{k}\right) \delta\left(\tau_{k}\right)= & \left(1+b_{k}\right)\left(\exp \left(\int_{\tau_{k-1}}^{\tau_{k}} a(t) d t\right) \delta\left(\tau_{k-1}+0\right)\right. \\
& \left.+\int_{\tau_{k-1}}^{\tau_{k}} r(s) \exp \left(\int_{s}^{\tau_{k}} a(t) d t\right) d s\right)
\end{aligned}
$$




$$
\begin{aligned}
= & \left(1+b_{k}\right)\left[\left(\left(1+b_{k-1}\right) \delta\left(\tau_{k-1}\right)+q_{k-1}\right) \exp \left(\int_{\tau_{k-1}}^{\tau_{k}} a(t) d t\right)\right. \\
& \left.+\int_{\tau_{k-1}}^{\tau_{k}} r(s) \exp \left(\int_{s}^{\tau_{k}} a(t) d t\right) d s\right] \geqslant 0 .
\end{aligned}
$$

Таким образом, в силу примера 3 и замечания $2 R(t) \subseteq \widetilde{X}(t)$. Следовательно, $R(t)=\widetilde{X}(t)$.

\section{Список литературы}

1. Панасюк А.И., Панасюк В.И. Асимптотическая оптимизация нелинейнштх систем управления. Минск: Изд-во БГУ, 1977.

2. Толстоногов A. А. Дифференциальные включения в банаховом пространстве. Новосибирск: Наука, 1986.

3. Kurzhanski A.B., Valyi I. Ellipsoidal calculus for estimation and control. Boston: Birkhäuser, 1997.

4. Овсеевич А.И. Экстремалњне свойства эллипсоидов, аппроксимирующих области достижимости // Проблемы управления и теории информации 1983 . Т. 12 . № 2 . C. $1237-1240$.

5. Черноусько Ф. Л. Оценивание фазового состояния динамических систем. Метод эллипсоидов. М.: Наука, 1988.

6. Дончев $A$. Системы оптимального управления: возмущения, приближения и анализ чувствительности. М.: Мир, 1987.

7. Плотников В.А., Плотников А.В., Витюк А.Н. Дифференциальные уравнения с многозначной правой частью. Асимптотические методы. Одесса: Астропринт, 1999.

8. Никольский M. С. Об одном методе аппроксимации множества достижимости для дифференциального включения // ЖВМиМФ. 1988. Т. 28. № 8. С. 1252-1254.

9. Veliov $V$. Second order discrete approximations to linear differencial inclusions // SIAM J. Numer. Anal. 1992. V. 29. P. 439-451.

10. Hukuhara $M$. Integration des applications mesurables dont la valeur est un compact convexe // Funkcial Ekvac. 1967. № 10. P. 205-223.

11. Тюрин Ю. Н. Математическая формулировка упрощенной модели производственного планирования // Эконом. и матем. методы. 1965. Т. 1. № 3. С. 391-409.

12. Banks H. T., Jacobs M. Q. A differential calculus of multifunctions // J. Math. Anal. Appl. 1970. № 29. P. 246-272.

13. Radström H. An embedding theorem for spaces of convex sets // Proc. Amer. Math. Soc. 1952. № 3. P. 165-169.

14. Половинкин E. С. Теория многозначных отображений. М.: Изд-во МФТИ, 1983.

15. Debreu G. Integration of correspondences // Proc. Fifth Berkeley Sympos. Math. Statist. and Probability (Berkeley, Calif., 1965/66). Vol. II: Contributions to Probability Theory, Part 1. Berkeley: Univ. California Press, 1967. P. 351-372.

16. de Blasi F. S., Iervolino F. Equazioni differentiali con soluzioni a valore compatto convesso // Boll. Unione Mat. Ital. 1969. V. 2. № 4-5. P. 491-501.

17. Aumann R. J. Integrals of set-valued functions // J. Math. Anal. Appl. 1965. V. 12. № 1 . P. 1-12.

18. Благодатских В.И., Филиппов А. Ф. Дифференциальные включения и оптимальное управление // Топология, обынновенные дифференциальные уравнения, динамические системы. М.: Наука, 1985. С. 194-252.

19. Markov S. M. Existence and uniqueness of solutions of the interval differential equation $X^{\prime}=F(t, X) / /$ C.R. Acad. Sci. Bulgar. 1978. V. 31. № 12. P. 1519-1522.

E-mail: talie@ukr.net 